\title{
Greater yogurt consumption is associated with increased bone mineral density and physical function in older adults
}

Osteoporosis is an increasingly common, chronic condition estimated to affect over 200 million individuals worldwide ${ }^{(1)}$ with $6 \%$ of men and $21 \%$ of women aged 50-84yrs affected in the EU alone ${ }^{(2)}$. Previous data from observational studies and randomised controlled trials (RCTs) have reported significant positive associations between dairy intakes and bone health ${ }^{(3,4)}$. In the current study, we examined the association between yogurt intakes and bone mineral density (BMD), biochemical markers of bone health and physical function measures in a large cohort of free-living older adults $(n=4,310$, age range $60-102$ yrs) from the Trinity, Ulster, Department of Agriculture (TUDA) Ageing Cohort study.

Bone measures included total hip, femoral neck and vertebral BMD with bone biochemical markers. Physical function measures included Timed Up and Go (TUG), Instrumental Activities of Daily Living Scale and Physical Self-Maintenance Scale. Total hip and femoral neck BMD in females were 3.1-3.9 \% higher among those with the highest yogurt intakes $(n=970)$ compared to the lowest $(n=1,109 ; P<0.05)$ as were the TUG scores $(-6.7 \% ; P=0.013)$. In males, tartrate-resistant acid phosphatase (TRAP 5b) concentrations were significantly lower in those with the highest yogurt intakes $(-9.5 \% ; P<0.0001)$. In females, yogurt intake was a significant positive predictor of BMD at all regions. Each unit increase in yogurt intake in females was associated with a $31 \%$ lower risk of osteopenia (OR $0.69 ; 95 \%$ CI $0.49-0.96 ; P=0.032$ ) and a $39 \%$ lower risk of osteoporosis $(\mathrm{OR} 0.61 ; 95 \%$ CI $0.42-0.89 ; P=0.012$ ) and in males, a $52 \%$ lower risk of osteoporosis (OR 0.48; $95 \%$ CI $0 \cdot 24-0.96 ; P=0.038$ ).

These findings provide evidence, from a well characterized cohort of older adults, that yogurt consumption is a positively associated with BMD and that increasing yogurt intake could offer a valuable and cost-effective strategy for maintaining bone health and reducing frailty in older adults. Future RCTs are required to investigate the efficacy of such approaches.

This research was funded by the Irish Department of Agriculture, Food and the Marine through the grants 07FHRIUCD1 ("JINGO" 2007-2013) and 13F407 ("JINGO-JPI"/"ENPADASI" 2014-2016) and from the Northern Ireland Department for Employment and Learning (DEL), Cross-Border Research and Development Programme: "Strengthening the all Island Research Base". Funding was also provided from The National Dairy Council (NDC) Ireland. The funding organizations had no role in the design and conduct, collection, management, analysis, and interpretation of the data, or in the preparation, review, or approval of this analysis.

1. Cooper C (1999) Epidemiology of osteoporosis. Osteoporos Int 9, 2-8.

2. Ström O, Borgström F, Kanis AJ, et al. (2011) Osteoporosis: Burden, healthcare provision and opportunities in the EU. Arch Osteoporos 6, 59-155.

3. Heaney RP. Dairy and bone health (2009) J Am Coll of Nutr 28, 82-90.

4. Dietary guidelines for Americans. Dietary Guidelines Advisory Committee Report. 6. U.S. Department of Health and Human Services and U.S. Department of Agriculture, U.S. Government; Washington DC: 2005. 